\title{
The Blind Locksmith
}

\section{Terry Trowbridge}

For the blind locksmith

jigsaw puzzles are prayers.

His retirement is spent fingering little

quadrangle keys with teeth on all four sides.

Jigsaw puzzles turn his tabletop into

flat five hundred piece worlds made out of keys freed from locks.

Five hundred keys that fit into each other.

A perfect plane of keys, properly fit

so that tumblers are superfluous.

The unseen picture is an inchoate tribute

to a lifetime of key cutting and lock setting.

The puzzle box sits on his knee

like five hundred rosary beads to enlighten him.

For the blind locksmith,

jigsaw puzzles are nightmares.

His dreams are filled with quadrangles that are

square locks with tumblers on all four sides.

He cannot read the box to know how boxboard

pieces will turn in his fingers, rotating left and right.

The nth frustration of a lifetime of skilled clicking,

he cannot remember if he held this one up to this one already.

After sorting, the edge pieces fence a rectangular promise

of a world of locks that fit locks but shut no doors.

There are countless setbacks that he will have to overcome.

The puzzle box sits open on his napping knee,

spelling a braille of uncertainty

over whether it is a bulwark against fading skills

or a bulwark against admitting the need for closure.

York University

Toronto, ON

Canada

e-mail: trowbridgeterry@gmail.com

Publisher's Note Springer Nature remains neutral with regard to jurisdictional claims in published maps and institutional affiliations. 